\title{
CROSS-SECTIONAL STUDY ON VACCINATION COVERAGE IN NEWLY DIAGNOSED HIV-INFECTED PERSONS IN THE CZECH REPUBLIC
}

\author{
David Jilich', Marek Malý2, Lukáš Fleischhans', Veronika Kulírová ${ }^{3}$, Ladislav Machala ${ }^{4}$ \\ 'Department of Infectious and Tropical Diseases, First Faculty of Medicine, Charles University and Na Bulovce Hospital, Prague, Czech Republic \\ ${ }^{2}$ Department of Biostatistics, National Institute of Public Health, Prague, Czech Republic \\ ${ }^{3} \mathrm{Na}$ Bulovce Hospital, Prague, Czech Republic \\ ${ }^{4}$ Department of Infectious Diseases, Third Faculty of Medicine, Charles University and Na Bulovce Hospital, Prague, Czech Republic
}

\section{SUMMARY}

Objectives: Individuals with HIV infection are at an increased risk for a number of infectious diseases, some of which are preventable by vaccination. Unfortunately, little is known about the attitudes of this population group to vaccination, therefore, we decided to find out vaccination coverage against 5 infections among newly diagnosed HIV-infected patients in the Czech Republic.

Methods: This cross-sectional study was conducted on newly diagnosed patients who started their follow-up care at the HIV Clinic of Na Bulovce Hospital during the two following years. Vaccination history data and results of serological tests were collected from all participants.

Results: Enrolled were 269 HIV-positive subjects ( $94.1 \%$ males) with a mean age of 34.4 years, 64 subjects (23.8\%) had tertiary education, 229 (85.1\%) were men having sex with men, $32(11.9 \%)$ were heterosexual, and $8(3.0 \%)$ were injection drug users. The mean CD4+ T-lymphocyte count was $556.2 / \mu \mathrm{L}$, with 149 persons $(55.4 \%)$ who had a CD4+ T-lymphocyte count $>500 / \mu \mathrm{L}$, and $68(25.3 \%)$ individuals were late presenters with CD4+ T-lymphocyte count $<350 / \mu \mathrm{L}$. A vaccination against tetanus was reported by 262 subjects $(97.4 \%)$, against influenza by 18 subjects (6.7\%), against tick-borne encephalitis by 18 subjects (6.7\%), against viral hepatitis $A$ by 78 persons (29.0\%), and against hepatitis B by 104 subjects (38.7\%). For influenza, tick-borne encephalitis and hepatitis $A$, a significant positive impact of tertiary education was found ( $p$-values $<0.001-0.044)$. Vaccination coverage against both types of hepatitis was significantly lower in late presenters $(p=0.044$ and $p=0.004$, respectively).

Conclusions: Vaccination rates found in our cohort were except tetanus and hepatitis B in young people low, especially for influenza and tick-borne encephalitis. Higher level of education and less advanced HIV infection were associated with higher vaccination rates. To improve this unsatisfactory situation, more attention should be paid to vaccination.

Key words: vaccination coverage, HIV, tetanus, tick-borne encephalitis, influenza, hepatitis

Address for correspondence: D. Jilich, Department of Infectious and Tropical Diseases, Na Bulovce Hospital, Budínova 48/2, 180 81, Prague 8, Czech Republic. E-mail: david.jlich@centrum.cz

https://doi.org/10.21101/cejph.a5830

\section{INTRODUCTION}

People living with human immunodeficiency virus (HIV) are increasingly vulnerable to a variety of infections, some of which are quite preventable by vaccination, but the effectiveness of vaccination programmes in HIV-infected individuals can be limited. The main concerns traditionally are about the efficacy and safety of vaccinations in this population group, and until now much less attention has been paid to issues such as vaccination coverage of people living with HIV or to their attitudes to various immunization programmes $(1,2)$.

In the Czech Republic (CR), there is only limited information about vaccination coverage of adults in the general population and data about the situation among HIV-infected individuals is completely missing $(3,4)$.

For this reason we decided to conduct a study with the goal to determine the vaccination coverage of $\mathrm{HIV}$-infected people living in this country. We have chosen 5 vaccination-preventable infectious diseases (tetanus, influenza, tick-borne encephalitis, hepatitis A and B). As these infections differ significantly in their epidemiology, there are also important differences in their vaccination strategies.

Tetanus is a very serious wound bacterial infection with a high mortality rate, but due to a long tradition of vaccination, its incidence in the CR in the past few decades has been very low (5). Vaccination against tetanus in the CR is mandatory by law and is completely covered by health insurance or health insurers. The national vaccination programme started in 1958 by the vaccination of all children at the age of three months and in 1975 regular vaccination of all adults over 18 years of age was introduced. The immunization against tetanus is based on administration of a specific toxoid and vaccination in the country is provided by both paediatricians and general practitioners (6). 
Influenza is the most common seasonal respiratory viral infection which significantly contributes to an increase of morbidity and mortality of immuno-compromised individuals but is preventable by vaccination (7). Vaccination against influenza in the $\mathrm{CR}$ is broadly offered to the general population and strongly recommended to groups with an increased risk of severe complications of flu as seniors over 65 years of age, persons with severe comorbidities inclusive of those with HIV infection. Vaccination against flu is not generally covered by health insurance in the CR but for healthcare workers the vaccination is usually sponsored by their employers. For immunization against flu are in the CR approved inactivated vaccines only.

Tick-borne encephalitis (TBE) is a typical vector-borne viral infection which can have serious neurological sequelae and its incidence in the CR is one of the highest in Europe (8). Vaccination against TBE in the country is widely recommended to all residing persons. For immunization against TBE in the $C R$ various inactivated vaccines are used and the vaccination is not covered by health insurance companies (9).

Viral hepatitis A and viral hepatitis B (HAV and HBV) are two types of liver infections with different epidemiology characterized by faecal-oral and parenteral transmission route, respectively.

For immunization against HAV and HBV in the CR are available inactivated vaccines either against single types or as a fixed combination of both.

The mandatory vaccination of three-month old infants against hepatitis B in the CR was implemented in 2001 together with a catch-up strategy for children at the age of 12 . This vaccination is covered by health insurance. However, with this age group HBV vaccination is routinely provided to persons at occupational risk, e.g. healthcare workers. The HBV vaccination is also generally recommended to individuals with risky sexual practices or drug addiction but without coverage by health insurance (10).

Vaccination against HAV in the CR is broadly recommended to the general population and particularly to individuals with increased risk like promiscuous sexual behaviour or drug abuse. This HAV vaccination is not covered by the health insurance system in the CR (11).

\section{MATERIALS AND METHODS}

\section{Study Population}

This cross-sectional study includes newly diagnosed patients with the HIV-1 infection who were over 18 years of age and initiated their follow-up care at a HIV clinic of $\mathrm{Na}$ Bulovce Hospital between 1 January 2014 and 31 December 2015. In the study all those who were able to provide vaccination history data and had related laboratory markers available were enrolled. The study was approved by the local ethics committee and informed consent was obtained from each enrolled patient.

Data was obtained during the first routine check-up. A questionnaire asking for the level of education and vaccination history including the type of vaccine, number of doses and timing of vaccination was used in all of them. In some patients further data was completed in an interview with their general practitioners (GPs) or by checking their standardized Certificate of Vaccination.
All vaccination history data except influenza were proved through serological tests. Samples were tested for IgG antibodies against tetanus toxoid using Elisa (R-Biopharm, Darmstadt, Germany), tick-borne encephalitis using Elisa method (Mast Diagnostica, Reinfeld, Germany) and for viral hepatitis using CMIA method (Abbott Laboratories, Abbott Park, IL) classified according to the manufacturer's protocol.

For tetanus, as positive vaccination history was taken from a vaccination scheme with at least three doses plus a booster dose within last fifteen years. As protective antibody titres were considered levels of specific IgG antibodies $>0.1 \mathrm{IU} / \mathrm{mL}$.

As vaccinated against influenza was considered by all subjects with history of at least one dose of vaccine within last fifteen years.

For TBE, as a positive vaccination history was considered with a vaccination scheme with at least three doses eventually plus a booster dose and as protective were accepted levels of specific IgG antibodies $>110 \mathrm{VU} / \mathrm{mL}$.

For HAV, as positive vaccination history was considered with a vaccination scheme with at least two doses and as protective were admitted levels of anti-HAV IgG $>15 \mathrm{mIU} / \mathrm{mL}$.

For $\mathrm{HBV}$, as positive vaccination history was taken with a vaccination scheme with at least three doses eventually plus a booster dose and as protective serologic titres were classified levels of anti-HBs IgG $>10 \mathrm{mIU} / \mathrm{mL}$.

\section{Statistical Analysis}

Continuous data is characterized by means and standard deviations and compared among the groups by a Student's t-test. Categorical data is presented as counts and percentages. Fisher's exact test and its generalization were used to analyze the differences in proportions among the groups and to assess the association between vaccination coverage and potential predictors including demographic and HIV-related factors. All statistical tests were evaluated as two-sided at a significance level of 0.05 . Statistical analysis was performed by the statistical software Stata, release 9.2 (Stata Corp LP, College Station, TX).

\section{Ethics Approval and Consent to Participate}

The Ethics Committee of Na Bulovce Hospital (Ref. No. IORG0006697) has reviewed and approved a research study "Investigation of the number of vaccinated patients with recently diagnosed HIV infection" based on the design of the study and draft of informed consent form on its regular meeting on 10 January 2017 (Reg. No. dated 6 January 2017/8325/EK-Z). Signed informed consent was obtained from every patient enrolled in the study.

\section{RESULTS}

\section{Cohort Characteristics}

Three hundred and seven people initiated their follow-up care as newly diagnosed at the HIV Clinic of Na Bulovce Hospital between 1 January 2014 and 31 December 2015.The enrolment process to the study was successful in 269 subjects (87.6\%).

The mean age at the time of HIV diagnosis was 34.4 years (standard deviation 9.2, range 19-72 years). As summarized in 
Table 1, more than three fifths $(63.2 \%)$ were in the age group of $25-39$ years. The vast majority $(253,94.1 \%)$ were males and $215(79.9 \%)$ participants were born in the CR. Sixty-four persons $(23.8 \%)$ had attained tertiary education, with the remaining 205 persons $(76.2 \%)$ having lower than tertiary education. Sexual contact was likely the route of acquiring HIV in 229 (85.1\%) men having sex with men (MSM) and in 32 (11.9\%) heterosexuals while 8 subjects $(3.0 \%)$ were intravenous drug users.

Mean CD4+ T-lymphocyte (CD4+) count was $556.2 / \mu \mathrm{L}$ (standard deviation 316.6 , range $6-1,700 / \mu \mathrm{L}$ ). One-hundred and forty-nine persons $(55.4 \%)$ had at the time of HIV-diagnosis CD4+ count higher than $500 / \mu \mathrm{L}$. Sixty-eight persons $(25.3 \%)$ were diagnosed as late presenters with CD4+ count lower than $350 / \mu$ including 38 persons (14.1\%) with advanced HIV-disease presenting CD4+ count lower than $200 / \mu \mathrm{L}$ (Table 1). In majority (75.0\%) of late presenters there was an absence of AIDS-defining symptoms. In 17 late presenters, however, HIV infection was diagnosed directly in the $\mathrm{C}$ stage with following AIDS defining diagnoses: 5 pneumocystis pneumonia, four oesophageal candidiasis, three lymphoma, two tuberculosis, two brain toxoplasmosis, and one HIV encephalopathy.

\section{Vaccination against Tetanus}

Vaccination against tetanus has been reported in 262 (97.4\%) subjects and all of them had protective IgG antibody titre (range $0.11-6.8 \mathrm{IU} / \mathrm{mL})$. The remaining 7 subjects $(2.6 \%)$ were not able to recall if they had been vaccinated against tetanus. In two of them serological tests were not done, whereas 5 individuals had protective IgG titres, so protective titres were altogether found in 267 subjects $(99.3 \%)$. No one had previously experienced tetanus disease.

Table 1. Basic characteristics of study cohort participants $(N=269)$

\begin{tabular}{|c|c|}
\hline Characteristic & $n(\%)$ \\
\hline Male gender & $253(94.1)$ \\
\hline Born in CR & $215(79.9)$ \\
\hline MSM & $229(85.1)$ \\
\hline Tertiary education & $64(23.8)$ \\
\hline CD4+ less than $350 / \mu \mathrm{L}$ & $68(25.3)$ \\
\hline CD4+ less than $200 / \mu \mathrm{L}$ & $38(14.1)$ \\
\hline
\end{tabular}

Following from Table 2, no statistically significant difference in reported vaccination cover $(\mathrm{p}=1.000)$ was observed among 64 subjects with tertiary education and 205 subjects with subtertiary education.

\section{Vaccination against Influenza}

Influenza vaccination was reported by 18 subjects $(6.7 \%)$, all being men, while 251 subjects $(93.3 \%)$ had never been vaccinated. Two subjects (11.1\% from all vaccinated) underwent influenza vaccination twice and 4 (22.2\% from all vaccinated) more than twice.

The proportion of influenza vaccinees was significantly higher $(p=0.003)$ among persons with tertiary education $(15.6 \%)$ than among those with sub-tertiary educational level (3.9\%) (Table 2). The vaccination coverage among late presenters $(4.4 \%)$ was lower than that among patients with less advanced HIV infection (7.5\%) but the difference was not significant. Likewise, no association of vaccination rates with age at HIV diagnosis was noticed.

\section{Vaccination against TBE}

Positive vaccination history against TBE was found in 18 subjects $(6.7 \%)$, all males. Negative vaccination history was found in 251 subjects (93.3\%). In all 18 vaccinated subjects, positive titres of IgG antibodies were found (range 150-1,600 $\mathrm{VU} / \mathrm{mL}$ ). None of them had declared TBE disease in the past. Among 251 unvaccinated subjects no seropositivity against TBE was detected.

Table 2 shows that there was a significantly higher proportion of vaccinated individuals among persons with tertiary $(12.5 \%)$ compared to that with sub-tertiary $(4.9 \%)$ education $(\mathrm{p}=0.044)$. The vaccination coverage of late presenters $(4.4 \%)$ was insignificantly lower than in patients with less advanced HIV infection (7.5\%). No association of vaccination rates with age at HIV diagnosis was seen.

\section{Vaccination against $\mathrm{HAV}$ and $\mathrm{HBV}$}

Positive vaccination history against HAV was reported by 78 subjects $(29.0 \%)$ while 191 subjects $(71.0 \%)$ were not vaccinated. All 78 vaccinated respondents had protective anti-HAV IgG titres (range from 16 to more than $1,000 \mathrm{mIU} / \mathrm{mL}$ ). Positive serology was also detected in $3(1.6 \%)$ unvaccinated persons even though none of them declared that they had undergone hepatitis $A$ in the past.

Table 2. Vaccination coverage according to level of education $(N=269)$

\begin{tabular}{|l|c|c|c|c|}
\hline \multirow{2}{*}{ Vaccination coverage } & \multicolumn{5}{|c|}{ Education } \\
\cline { 2 - 5 } & $\begin{array}{c}\text { Total } \\
(\mathrm{N}=\mathbf{2 6 9})\end{array}$ & $\begin{array}{c}\text { Tertiary } \\
(\mathbf{n = 6 4 )}\end{array}$ & $\begin{array}{c}\text { Sub-tertiary } \\
(\mathbf{n}=\mathbf{2 0 5})\end{array}$ & $\mathrm{p}$-value \\
\hline Tetanus & $262(97.4)$ & $63(98.4)$ & $199(97.1)$ & 1.000 \\
\hline Influenza & $18(6.7)$ & $10(15.6)$ & $8(3.9)$ & 0.003 \\
\hline TBE & $18(6.7)$ & $8(12.5)$ & $10(4.9)$ & 0.044 \\
\hline HAV & $78(29.0)$ & $31(48.4)$ & $47(22.9)$ & $<0.001$ \\
\hline HBV & $104(38.7)$ & $30(46.9)$ & $74(36.1)$ & 0.142 \\
\hline
\end{tabular}

Data are given as count (\%) 
Positive vaccination history against HBV was reported by 104 subjects (38.7\%). Negative vaccination history was given by 165 subjects $(61.3 \%)$. Protective titres of IgG anti-HBs (range from 11 to more than $1,000 \mathrm{mIU} / \mathrm{mL}$ ) were detected in $85(81.7 \%)$ of vaccinated individuals whereas the remaining 19 had the titres lower than protective limit. Four unvaccinated subjects declared that they undergone hepatitis B disease in the past.

The majority of participants vaccinated against viral hepatitis was vaccinated against both infections (60 simultaneously and 5 separately in different times). These 65 persons $(24.2 \%$ of the cohort) make up a proportion of $83.3 \%$ of all HAV vaccinees and $62.5 \%$ of all $\mathrm{HBV}$ vaccinees. Positive vaccination history against HAV only was reported by 13 subjects (4.8\%) and against HBV only by 39 subjects $(14.5 \%)$.

We found that there was a positive impact of tertiary education on vaccination coverage (Table 2). Subjects with tertiary education were more likely vaccinated against HAV than subjects with lower education $(48.4 \%$ vs. $22.9 \%$ ), p $<0.001$. For HBV vaccination the difference was in the same direction but less pronounced $(46.9 \%$ vs. $36.1 \%)$ and did not reach statistical significance $(p=0.142)$.

Significantly higher HBV vaccination coverage rate was noticed among 44 subjects in age group of 18-26 years (84.1\%) compared to 225 older subjects $(29.8 \%), \mathrm{p}<0.001$. Particularly high coverage was seen in the above-mentioned younger age group of Czech patients (94.1\%). No such difference was found for HAV where vaccination rates in both age groups were similar ( $27.3 \%$ vs. $29.3 \%), p=0.857$.

Vaccination coverage against both HAV and HBV was significantly higher among those with less advanced HIV infection at the moment of diagnosis compared to late presenters. Corresponding rates are $32.3 \%$ and $19.1 \%$, respectively, for $\operatorname{HAV}(\mathrm{p}=0.044)$, and $43.8 \%$ and $23.5 \%$, respectively, for HBV $(p=0.004)$.

\section{Vaccination against Multiple Non-mandatory Infections}

Less than one half $(130,48.3 \%)$ of analyzed subjects underwent vaccination against at least one of four investigated infections (influenza, TBE, HAV, HBV) for which it is recommended but voluntary in the Czech Republic. Out of them, 55 individuals were vaccinated against the only infection (8 against influenza, four against TBE, 9 against $\mathrm{HAV}$, and 34 against $\mathrm{HBV}$ ), 62 participants against two infections (54 against HAV+HBV, one against influenza + TBE 1, two against influenza $+\mathrm{HAV}$, two against influenza $+\mathrm{HAV}$, two against TBE $+\mathrm{HBV}$, and one against TBE $+\mathrm{HAV}$ ), and 13 patients against three infections (three against influenza $+\mathrm{HAV}+\mathrm{HBV}$, eight against TBE + HAV + HBV, one against influenza + TBE + HAV, and one against influenza $+\mathrm{TBE}+\mathrm{HAV}$ ). No one was vaccinated against all four infections preventable by voluntary vaccination. Participants with tertiary education were more likely to be voluntarily vaccinated against at least one infection than participants with lower education $(68.8 \%$ vs. $42.0 \%, \mathrm{p}<0.001)$. Vaccination against two or three infections was reported by $45.3 \%$ of subjects with tertiary education but only by $22.4 \%$ of subjects with sub-tertiary education.

\section{DISCUSSION}

Our cohort was made up of a total proportion of $54.0 \%$ of all persons diagnosed with HIV infection in the same period
(2014-2015) in the CR. The age, gender and way of transmission of the study participants are fully consistent with the entire Czech situation and thus our cohort is representative for the whole country (12).

\section{Vaccination against Tetanus}

The vaccination coverage self-reported by patients $(97.4 \%)$ corresponds with the general situation in the country with a long tradition of mandatory vaccination (13). Moreover, the positive serology in 5 individuals, who were not able to remember the vaccination, can be explained only as evidence of a successful vaccination done in the past. Thus, in reality at least $99.3 \%$ was vaccinated in our cohort, because with the extremely low incidence of tetanus in the CR it is probable that those antibodies were not consequence of a real tetanus infection (5). Though this high vaccination coverage without any differences in age or education of vaccinated individuals illustrates well-functioning system of mandatory vaccination against tetanus (13).

\section{Vaccination against Influenza}

The very low influenza vaccination coverage of only $6.7 \%$ observed in our cohort is unsurprising and fully consistent with the general Czech population showing vaccination cover rates of $5.0 \%$ in the season $2013 / 14$, and $4.9 \%$ in the season $2014 / 15$ (14, 15). Overall, the CR thus ranks among the other Central-Eastern European countries with the lowest level of influenza vaccination coverage rates (below 10\%) like Poland (general population 9.5\%) or Estonia and Latvia with only $1 \%$ in the age group $\geq 65$ years $(16,17)$. On the other hand, in Western European countries the vaccination coverage is typically over $30 \%$ (18).

To explain the reasons for unsatisfactory vaccination coverage rates in Central and Eastern European countries is not easy. The factors which may play a major role include general lack of accurate information about influenza and thus a low perception of risk and fear of possible and perceived side effects from vaccination, misleading reports in the mainstream media, broader anti-vaccine sentiments, and also issues of cost which may play important role particularly in lower-income-countries (19).

As in other studies, however, a higher level of education showed an unambiguously positive effect on the attitude to flu vaccination with four-times higher coverage in the subgroup of tertiary-educated subjects $(p=0.003)(20)$.

\section{Vaccination against Tick-borne Encephalitis}

The vaccination coverage rate of $6.7 \%$ found in our cohort is low compared with the general population in the country. In various studies in the $\mathrm{CR}$, the vaccination rates range between $15 \%$ in adults and $22 \%$ in school-aged children $(8,21)$. Even in neighbouring countries with similar epidemiological situation vaccination coverage is very different. For example, in Austria the vaccination rate is around $85 \%$, in Slovenia $12 \%$ and in Poland is as low as $0.8 \%(22-24)$. Explanation for such low and unsatisfactory vaccination cover rates in post-communist countries of Central-Eastern Europe despite the unfavourable epidemiological situation could be persistent low disease awareness, and the same with relatively high costs of vaccines. This hypothesis could be 
supported by higher vaccination cover rates in persons with higher level of education as similarly seen in influenza $(25,26)$.

Positive serology in all vaccinated individuals is highly probably attributable to the vaccination, however, the possibility of latent TBE infection before the vaccination cannot be excluded. The asymptomatic TBE infections are very common and for example in the CR was found the prevalence of specific TBE antibodies in around $25 \%$ of population that had never have any symptoms of TBE (27).

On the other hand, the complete absence of TBE antibodies among non-vaccinated patients can be explained by low probability of exposure because of completely different epidemiology of HIV and TBE. The HIV infection is a typically urban disease and TBE is a typically rural infection. These differences make co-infection of these very unlikely similarly as seen in co-infection between HIV and Lyme disease (28).

\section{Vaccination against Viral Hepatitis A and Hepatitis B}

Most of published vaccination studies on viral hepatitis have been focused on immunogenicity and the efficacy of vaccination whereas the vaccination coverage assessment has been paid little attention and so there is not much data available. Vaccination coverage against HAV (29.0\%) and HBV (38.7\%) in our cohort is relatively low compared with that found among HIV-positive individuals in other countries, e.g. in France $61.9 \%$ and in Brazil with $57.4 \%$ or $76.9 \%$, respectively (29-31).

Not surprisingly a substantially higher coverage rate against HBV was found in the age group below 26 years. This finding corresponds with the introduction of national catch-up strategy among 12-year-olds since 2001 (3). As a consequence of the mandatory vaccination against $\mathrm{HBV}$, the education-based difference in vaccination coverage is less pronounced for this infection, whereas for completely non-mandatory HAV the vaccination coverage not surprisingly increases with educational level.

The higher vaccination coverage against both HAV and HBV among individuals who were diagnosed as HIV in less advanced stage may be explained by their higher interest in the health status and their higher perception of health risks in conjunction with the effect of vaccination promotion campaigns among groups with risky behaviour (32). Very low seropositivity rate of anti-HAV antibodies among non-vaccinated individuals in our cohort is consistent with most recently published serological survey from the region, where the overall prevalence all antiHAV positive individuals regardless vaccination history was $37.4 \%$ (33). Based on this, the Czech Republic does not belong to low-prevalence HAV countries with higher prevalence among HIV-positive individuals compared to HIV-negative population $(34,35)$.

\section{Vaccination against Multiple Non-mandatory Infec- tions}

Significantly higher vaccination coverage against multiple infections in the group with higher education $(45.3 \%$ vs. $22.4 \%$ ) is also not surprising because individuals with higher education usually pay more attention to their health status and thus have also better life-expectancy as seen in the Swiss HIV cohort (36).

\section{CONCLUSIONS}

The results of our study have shown significant differences in vaccination coverage against analyzed infections. The highest coverage was not surprisingly against tetanus and HBV in people younger than 26 . The vaccination coverage against other infections was very low, particularly in case of TBE and influenza. Clearly, a positive effect on the vaccination coverage depends on the level of education but even in the group of highly educated individuals the vaccination coverage with non-mandatory vaccinations is unsatisfactorily low. The explanation in general can probably be seen in very low disease awareness, insufficient social marketing, low support by general practitioners, as well as increasing trends of anti-vaccination tendencies in our region (37).

To improve this unsatisfactory situation, it is necessary to pay on-going attention to all vaccination issues in people with high risk behaviour in terms of HIV infection including further research of those factors which influence attitudes to vaccination in those difficult to reach population groups.

\section{Acknowledgements}

Authors would like to thank all the patients participating in the study as well as the whole research team.

\section{Conflict of Interests}

None declared

\section{REFERENCES}

1. Crum-Cianflone NF, Wallace MR. Vaccination in HIV-infected adults. AIDS Patient Care STDS. 2014;28(8):397-410.

2. Blackwell CW. Knowledge of vaccination needs of HIV-infected men who have sex with men in a national sample of "gay friendly" health care providers. Public Health Nurs. 2016;33(5):403-11.

3. Dlhý J, Štěpánková H. Administrative control of vaccination coverage in the Czech Republic by December 31, 2009. Epidemiol Mikrobiol Imunol. 2011;60(2):55-63. (In Czech.)

4. Danova J, Kocourkova A, Celko AM. Active surveillance study of adverse events following immunisation of children in the Czech Republic. BMC Public Health. 2017;17(1):167. doi: 10.1186/s12889-017-4083-4.

5. European Centre for Disease and Control. Anual epidemiological report 2014: vaccine preventable diseases. Stockholm: ECDC; 2014.

6. Matoušková I, Kollárová H, Janoutová G, Janout V. Vaccination against tetanus. Vakcinologie. 2007;1(3):130-9. (In Czech.)

7. Sheth AN, Althoff KN, Brooks JT. Influenza susceptibility, severity, and shedding in HIV-infected adults: a review of the literature. Clin Infect Dis. 2011;52(2):219-27.

8. Kriz B, Maly M, Benes C, Daniel M. Epidemiology of tick-borne encephalitis in the Czech Republic 1970-2008. Vector Borne Zoonotic Dis. 2012;12(11):994-9.

9. Zavadska D, Anca I, Andre F, Bakir M, Chlibek R, Cizman M, et al Recommendations for tick-borne encephalitis vaccination from the Central European Vaccination Awareness Group (CEVAG). Hum Vaccin Immunother. 2013;9(2):362-74.

10. World Health Organization. Immunization of people living with HIV and people at risk of HIV infection: clinical protocol for the WHO European Region [Internet]. [cited 2019 Aug 20]. Available from: http://www.euro. who.int/_data/assets/pdf_file/0004/78502/E90840_Chapter_12.pdf.

11. Krausová J, Kosina P. Vaccination in adults - general principles. Med Praxi. 2013;10(4):142-5. (In Czech.)

12. Malý M, Němeček V, Zákoucká $H$. The prevalence and spread of HIV/ AIDS in the Czech Republic in 2015. Zpravy CEM. 2016;25(9-10):32030. (In Czech.)

13. World Health Organization. National Immunization Coverage Scorecards. Geneva: WHO; 2015. 
14. Puig-Barbera J, Burtseva E, Yu H, Cowling BJ, Badur S, Kyncl J, et al. Influenza epidemiology and influenza vaccine effectiveness during the 2014-2015 season: annual report from the Global Influenza Hospital Surveillance Network. BMC Public Health. 2016;16 Suppl 1:757. doi: 10.1186/s12889-016-3378-1.

15. Kynčl J, Havlíčková M. Influenza is a preventable disease. Med Praxi. 2013;10(8-9):279-81. (In Czech.).

16. Mereckiene J, Cotter S, Nicoll A, Lopalco P, Noori T, Weber J, et al. Seasonal influenza immunisation in Europe. Overview of recommendations and vaccination coverage for three seasons: pre-pandemic (2008/09), pandemic (2009/10) and post-pandemic (2010/11). Euro Surveill. 2014;19(16). doi: 10.2807/1560-7917.ES2014.19.16.20780.

17. Blank PR, Schwenkglenks M, Szucs TD. Vaccination coverage rates in eleven European countries during two consecutive influenza seasons. J Infect. 2009;58(6):446-58.

18. Schmid P, Rauber D, Betsch C, Lidolt G, Denker ML. Barriers of influenza vaccination intention and behavior - a systematic review of Influenza vaccine hesitancy, 2005 - 2016. PLoS One. 2017;12(1):e0170550. doi: 10.1371/journal.pone.0170550.

19. Böhmer MM, Walter D, Falkenhorst G, Muters S, Krause G, Wichmann O. Barriers to pandemic influenza vaccination and uptake of seasonal influenza vaccine in the post-pandemic season in Germany. BMC Public Health. 2012;12:938. doi: 10.1186/1471-2458-12-938.

20. Nagata JM, Hernandez-Ramos I, Kurup AS, Albrecht D, Vivas-Torrealba C, Franco-Paredes C. Social determinants of health and seasonal influenza vaccination in adults $>/=65$ years: a systematic review of qualitative and quantitative data. BMC Public Health. 2013;13:388. doi: 10.1186/14712458-13-388

21. Cabrnochová H, Skibová J. Child vaccination rate in the Czech Republic those that apply to optional vaccination. Vakcinologie. 2010;4(2):50-4. (In Czech.)

22. Heinz FX, Stiasny K, Holzmann H, Grgic-Vitek M, Kriz B, Essl A, et al. Vaccination and tick-borne encephalitis, Central Europe. Emerg Infect Dis. 2013;19(1):69-76.

23. Kollaritsch H, Chmelik V, Dontsenko I, Grzeszczuk A, Kondrusik M, Usonis V, et al. The current perspective on tick-borne encephalitis awareness and prevention in six Central and Eastern European countries: report from a meeting of experts convened to discuss TBE in their region. Vaccine. 2011;29(28):4556-64.

24. Grgic-Vitek M, Klavs I. Low coverage and predictors of vaccination uptake against tick-borne encephalitis in Slovenia. Eur J Public Health. 2012;22(2):182-6.

25. Prymula R, Prymulova K. How can we improve compliance with vaccination in Europe? Future Microbiol. 2016;10(9):1397-400.
26. Askling HH, Insulander M, Hergens MP, Leval A. Tick borne encephalitis (TBE)-vaccination coverage and analysis of variables associated with vaccination, Sweden. Vaccine. 2015;33(38):4962-8.

27. Kriz B, Hubalek Z, Marek M, Daniel M, Strakova P, Betasova L. Results of the screening of tick-borne encephalitis virus antibodies in human sera from eight districts collected two decades apart. Vector Borne Zoonotic Dis. 2015;15(8):489-93.

28. Garcia-Monco JC, Frey HM, Villar BF, Golightly MG, Benach JL. Lyme disease concurrent with human immunodeficiency virus infection. Am J Med. 1989;87(3):325-8.

29. Ho YL, Enohata T, Lopes MH, De Sousa Dos Santos S. Vaccination in Brazilian HIV-infected adults: a cross-sectional study. AIDS Patient Care STDS. 2008;22(1):65-70.

30. Valour F, Cotte L, Voirin N, Godinot M, Ader F, Ferry T, et al. Vaccination coverage against hepatitis A and B viruses, Streptococcus pneumoniae, seasonal flu, and A(H1N1)2009 pandemic influenza in HIV-infected patients. Vaccine. 2014;32(35):4558-64.

31. Martins S, Livramento A, Andrigueti M, Kretzer IF, Machado MJ, Spada $\mathrm{C}$, et al. Vaccination coverage and immunity against hepatitis B among HIV-infected patients in South Brazil. Braz J Infect Dis. 2015;19(2):181-6.

32. Matthews JE, Stephenson R, Sullivan PS. Factors associated with selfreported HBV vaccination among HIV-negative MSM participating in an online sexual health survey: a cross-sectional study. PLoS One. 2012;7(2):e30609. doi: 10.1371/journal.pone.0030609.

33. Němeček V, Částková J, Fritz P. Linhartová A, Švandová E, Šrámová H, et al. The 2001 serological survey in the Czech Republic - viral hepatitis. Cent Eur J Publ Health. 2003 Dec;11 Suppl:S54-61.

34. Nadwani R, Caswell S, Boag F, Lawrence AG, Coleman JC. Hepatitis A seroprevalence in homosexual and heterosexual men. Genitourin Med. 1994;70(4):325-8.

35. Sun HY, Kung HC, Ho YC, Chien YF, Chen MY, Sheng WH, et al. Seroprevalence of hepatitis A virus infection in persons with HIV infection in Taiwan: implications for hepatitis A vaccination. Int J Infect Dis. 2009;13(5):e199-205.

36. Gueler A, Moser A, Calmy A, Gunthard HF, Bernasconi E, Furrer H, et al. Life expectancy in HIV-positive persons in Switzerland: matched comparison with general population. AIDS. 2017;31(3):427-36.

37. Mad'ar R. Anti-vaccination activities in the Czech Republic in relation to the integrity of the vaccination schedules. Vakcinologie. 2013;7(3):122-9. (In Czech.) 